\title{
Recent results on Ne and Mg from the MISTRAL mass measurement program at ISOLDE
}

\author{
D. Lunney ${ }^{\mathrm{a}, *}$ C. Monsanglant ${ }^{\mathrm{a}}$ G. Audi ${ }^{\mathrm{a}}$ G. Bollen ${ }^{\mathrm{b}, * *}$ C. Borcea ${ }^{\mathrm{b}, \mathrm{c}}$ \\ H. Doubre ${ }^{a}$ C. Gaulard ${ }^{a}$ S. Henry ${ }^{a}$ M. de Saint Simon ${ }^{a}$ C. Thibault ${ }^{a}$ \\ C. Toader ${ }^{a, c}$ N. Vieira ${ }^{a}$ and the ISOLDE Collaboration ${ }^{b}$ \\ ${ }^{a}$ Centre de Spectrométrie Nucléaire et de Spectrométrie de Masse (CSNSM) \\ IN2P3-CNRS, Université de Paris Sud, Bât. 108, F-91405 Orsay, France \\ ${ }^{b}$ CERN, CH-1211, Geneva, Switzerland \\ ${ }^{c}$ INPE, Bucharest-Magurele, Romania
}

\begin{abstract}
The MISTRAL experiment (Mass measurements at ISOLDE with a Transmission and Radiofrequency spectrometer on-Line), conceived for very short-lived nuclides, has reached the end of its commissioning phase. Installed in 1997, results have been obtained consistent with all aspects of the projected spectrometer performance: nuclides with half-lives as short as $30 \mathrm{~ms}$ have been measured and accuracies of 0.4 ppm have been achieved, despite the presence of a systematic shift and difficulties with isobaric contamination. Masses of several nuclides, including ${ }^{25-26} \mathrm{Ne}$ and ${ }^{32} \mathrm{Mg}$ that forms the famous island of inversion around $N=20$, have been significantly improved.
\end{abstract}

\section{Introduction}

The determination of atomic masses suffers from two particular difficulties: the fact that masses are often determined from a series of reaction and/or decay values and the requirement of very high measurement precision. The former is the problem of the evaluator, such as explained by G. Audi in these proceedings, but can be helped by so-called "direct" methods of mass spectrometry. The second is the problem of the experimenter who must develop a reliable technique. This difficulty is compounded for studies of exotic nuclei where the half-lives become very short and the production, very weak.

Among several complementary direct techniques (see review by A. LépineSzily in these proceedings) one that is particularly attractive and has now delivered on its promise for precision measurements of short-lived nuclides is that exploited by MISTRAL, installed at the ISOLDE isotope separator facililty at CERN in 1997.

\footnotetext{
* e-mail address: lunney@csnsm.in2p3.fr
}

** now at NSCL, Michigan State University 


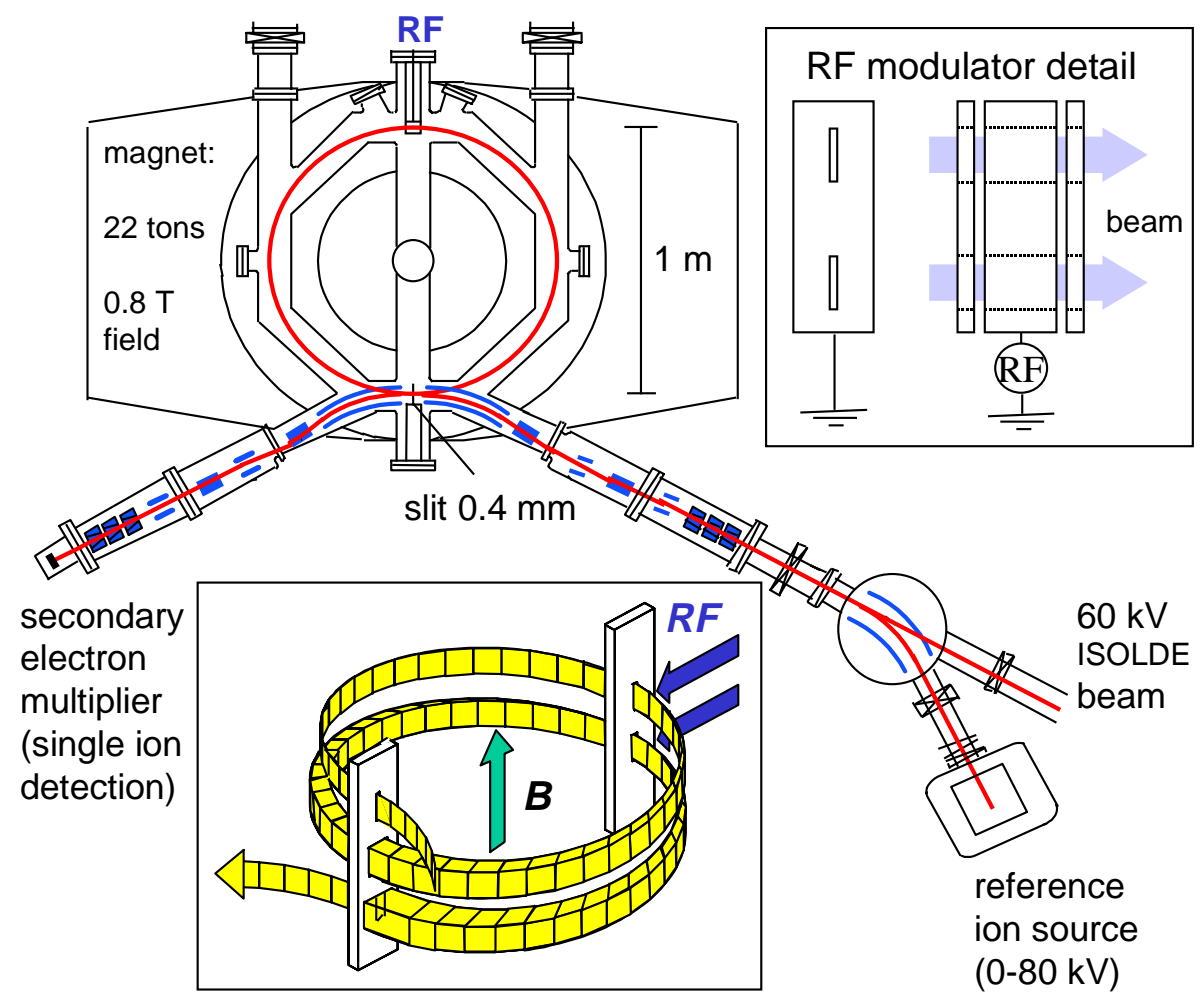

Figure 1. Overview of the MISTRAL spectrometer at ISOLDE. Insets show (lower left) the trajectory envelope and (upper right) front and side views of the modulator.

At once a magnetic mass spectrometer and a time of flight spectrometer, MISTRAL is a unique instrument. It was inspired by the original spectrometer conceived and built by L.G. Smith [1] in the 1960's. The main difference is that for use with weak (e.g., radioactive) beams, the very small slit that Smith used to define the ion trajectories must be enlarged.

MISTRAL determines the mass $m$ of an ion of charge $q$ in a homogeneous magnetic field $B$ by measuring its cyclotron frequency $f_{c}=\frac{1}{2 \pi} \frac{q}{m} B$ from transmission peaks produced by a radiofrequency voltage that modulates the kinetic energy of the injected $(60 \mathrm{keV})$ ISOLDE beam. The technique is rapid, requiring only the time-of-flight of the ions through the apparatus at full transport energy. It is also very precise since it is capable of very high resolving power $\left(>10^{5}\right)$, the magnetic field fluctuations are small and comparisons with a reference mass are performed very frequently to eliminate field drift effects. A layout of the spectrometer, together with the envelope of the modulated, isochronous ion trajectories, is shown in Fig. 1. For detailed descriptions see [2,3]. 


\section{The frequency spectrum of the transmitted ions}

Given a nominal magnetic field for ions at a given energy, the detected ion signal intensity will correspond to the fraction of the ISOLDE beam intensity determined by the geometrical acceptance (roughly $8 \pi \mathrm{mm} \mathrm{mrad}$ vertical and $2 \pi \mathrm{mm}$ mrad horizontal) divided by the incoming beam emittance (for $60 \mathrm{keV}$ ISOLDE beams, of the order of $35 \pi \mathrm{mm} \mathrm{mrad}$ or more in each transverse coordinate). A peak is obtained by recording the number of ions transmitted as a function of modulation frequency $f_{R F}$. In order to be transmitted, the relation $f_{R F}=\left(n+\frac{1}{2}\right) f_{c}$ must hold where $n$ is an integer harmonic (typically a few thousand). The radioactive beam from ISOLDE is injected into the spectrometer at alternating intervals with a reference ion source beam. In order not to change the magnetic field, the reference ion source voltage is tuned to keep the mass-voltage product constant. However we are still required to switch all the electrostatic optics voltages to transport the two beams through the same field.

Fig. 2 shows a modulation frequency scan for various radiofrequency (RF) voltages. When the RF voltage is turned on, troughs will start to appear where the above relationship is not respected. As the RF voltage increases, transmission peaks form. When the voltage is high enough, the amplitude of the ions' excursion from the nominal trajectory becomes larger than the opening of the phase definition slit, located at mid-turn, and some of the ions are blocked. The peak width narrows as a result with the wings being totally suppressed (with a price to pay in transmission, naturally). However there is still an area between two peaks that corresponds to an integer multiple of the cyclotron frequency over which a small number of ions are transmitted. This "peak" is not interesting for mass measurements due to its broad and flat form and worse, for intense isobars it is an important source of background. (A rigorous treatment of the detected ion signal is given in [4].) The curves in Fig.2 were generated by a Monte Carlo simulation that reproduces measured mass peaks with excellent agreement.

The resolving power of the spectrometer is inversely proportional to the exit slit size, proportional to the harmonic number of the cyclotron frequency and also to the modulation amplitude (the maximal radial excursion from the central trajectory), determined by two factors: how much voltage is applied to the modulator electrode and the modulation efficiency. The first factor depends on the frequency response of the circuit used to match the modulator impedance to the signal source, shown in Fig. 3 (upper left). The realization of a matching circuit over such a large frequency range is far from trivial [5]. The second factor, shown in Fig. 3 (lower left), is kinematic, varying with frequency and depending on ion velocity and modulator geometry. The ion sees two electric field gaps on each passage through the modulator (see Fig. 1 inset). For maximum modulation

efficiency, the ion velocity must be such that the field changes polarity during its flight through the central drift electrode to receive two tandem accelerations. If the velocity is such that the ion feels equal and opposite electric fields, the 


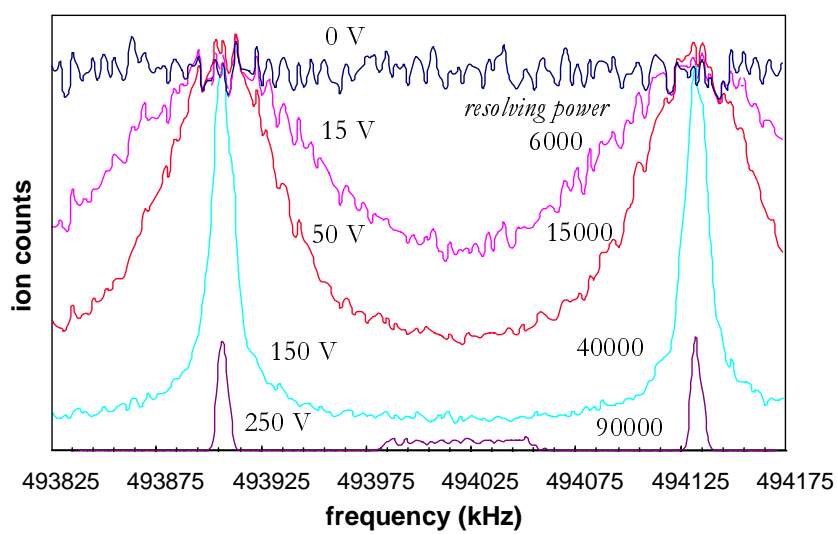

Figure 2. Transmitted $60 \mathrm{keV}^{23} \mathrm{Na}$ ion signal as a function of excitation frequency for various $\mathrm{RF}$ voltages and the resulting resolving power (generated by simulation).

net acceleration (and resulting modulation efficiency) is zero. As seen from the figure, this effect is periodic as the modulation frequency can go through many oscillations for slow ions. The efficiency diminishes with frequency since the net accelerating field is lowered for wider phase windows [5]. The modulation amplitude, the experimentally observable quantity, is the convolution of the above two factors. A radial profile of a modulated beam as a function of voltage is shown in Fig. 3 (right) with the modulation amplitude given by the position of the maximum intensity. (For mechanical reasons, it is not possible to measure the entire profile which is normally symmetric.)

In the case of isobaric contaminations it is not only resolving power that is important but also the relative position of the different peaks. A weak isobar can be very nicely resolved but if it falls inside the flat, integer peak of another isobar produced with greater abondance (e.g., a stable species several orders of magnitude stronger), we must change the frequency range in order to move the relative spacing of the two peaks. It can also happen that the mass difference of two isobars is large enough to be resolved and the two mass peaks still overlap but with different harmonic numbers. Again, a change in frequency is required to change the relative spacings. In cases where several isobars are present, this manoeuver - because of the limited range of modulation efficiency coupled with frequency response (Fig.3) - can easily become impossible.

\section{Mass measurements of $\mathrm{Mg}$ and $\mathrm{Ne}$ isotopes}

The first series of measurements with MISTRAL were performed using a surface ionization source that provides relatively pure beams. This way, the series ${ }^{23-30} \mathrm{Na}$ was measured [6]. The only isobaric contaminant encountered along the way was ${ }^{27} \mathrm{Al}$. As it turned out, while the mass difference between the 

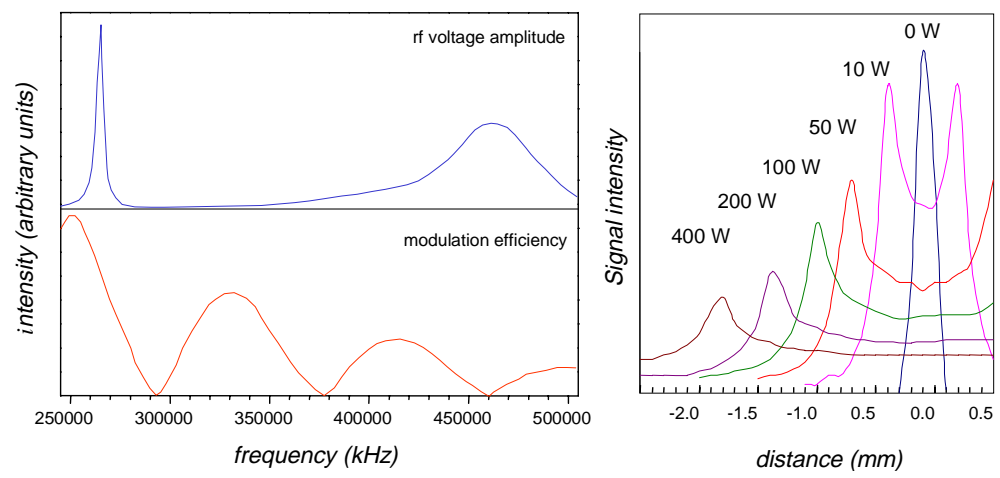

Figure 3. (upper left) The frequency response of the (tuned) impedance-matching circuit of the modulator. Though a flat response over the $250-500 \mathrm{MHz}$ frequency band is sought, only two resonances are achieved. The response is measured by an antenna mounted near the modulator having its own frequency response which is roughly deconvoluted. (lower left) The modulation efficiency for ${ }^{85} \mathrm{Rb}$ ions at $60 \mathrm{keV}$, computed by the Monte Carlo simulation as in fact, it is not accessible experimentally. The absolute amplitude for a given frequency is the product of these two quantities. (right) The $254 \mathrm{MHz}$ modulation amplitude of a $60 \mathrm{keV}{ }^{23} \mathrm{Na}$ beam measured at the spectrometer midplane.

two isobars is quite large, it corresponded to exactly one harmonic number at the frequency where we were working.

To gain more experience with the spectrometer, notably in handling isobaric contamination, we performed two runs with a plasma source. Coupled to a uranium carbide target by a heated transfer line, the plasma is the universal source for ISOLDE beams. Such a source permits the study of several chemical species during one run but has the great disadvantage of producing prodigious isobaric contamination. Not only are radiogenic species ionized but also elements constituting the source itself and worse, all manner of chemical molecules. Furthermore, multiple charge states are also present.

Several nuclides having well known masses were measured in order to evaluate the accuracy of the spectrometer. These results in fact revealed a systematic deviation of the mass value proportional to the mass difference [6]. In an attempt to calibrate this effect, two strategies were used: (1) a linear fit was made to the several measured calibrant masses (giving a slope) and combined with several measurements where the reference mass and the ISOLDE mass were the same $\left({ }^{23} \mathrm{Na}\right.$ - giving the offset) and (2) ISOLDE masses were measured with respect to two MISTRAL reference masses $\left({ }^{39 / 41} \mathrm{~K}\right)$ to give the slope of the calibration law. The latter technique has the advantage of providing the slope independantly of the ISOLDE mass (and its uncertainty).

It was necessary to use $\mathrm{K}$ as a reference to measure masses beyond $A=30$ since the voltage of the MISTRAL ion source is not sufficient for the lighter Na. Unfortunately, of the four calibrant masses measured using K, two were contaminated by unknown isobars. Moreover, very few like-mass comparisons 


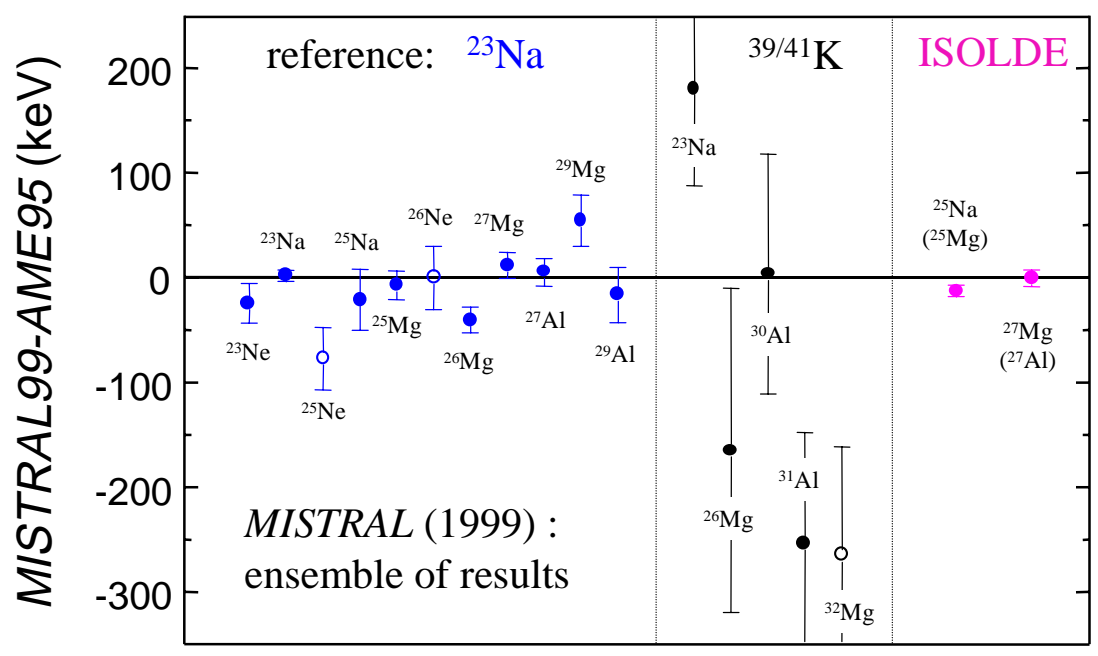

Figure 4. Absolute difference of MISTRAL masses compared to the 1995 mass evaluation [8]. The results are divided into three groups corresponding to the reference isotope used: (left) ${ }^{23} \mathrm{Na}$, (center) ${ }^{39} \mathrm{~K}$, (right) isobar in the ISOLDE beam, indicated in parentheses. The resulting dispersions of the calibrant masses (full symbols) are respectively, $1.1 \cdot 10^{-6}, 7.7 \cdot 10^{-6}$ and $3.5 \cdot 10^{-7}$.

were made using the $\mathrm{K}$ isotopes and their dispersion, for reasons we have yet to identify, is very large. In order for the values of the calibration parameters to have consistency, it was necessary to add a systematic error of $1.2 \cdot 10^{-6}$. The reader is refered to [7] for a discussion of the calibration procedure and detailed presentation of the results which are summarized here.

The results of the ensemble of nuclides measured is shown in Fig. 4 as the absolute difference with respect to the 1995 atomic mass evaluation [8]. On the left side of Fig. 4 are several nuclides whose masses were measured using ${ }^{23} \mathrm{Na}$ as the reference isotope. The calibrant masses are reproduced fairly well (with an average accuracy of $8 \cdot 10^{-7}$ ) and new values with reduced errors were obtained for two short-lived neon isotopes ${ }^{25-26} \mathrm{Ne}$.

In the center of fig. 4 are shown those nuclides measured with respect to ${ }^{39} \mathrm{~K}$ and/or ${ }^{41} \mathrm{~K}$. The larger error bars and dispersion in this case reflects the results of the less than satisfactory calibration. Despite the accuracy of only $3.5 \cdot 10^{-6}$ achieved for ${ }^{32} \mathrm{Mg}$ (also compounded by statistical error) it was still possible to improve the mass value for this exotic nuclide.

But why not turn the isobaric problem around and use it to our advantage? Provided we can identify them, isobars practically represent the closest thing to a narrow doublet, free from the deleterious effects of a large voltage jump. Indeed, the groups that are breaking the records of mass measurement precision (see the papers of S. Rainville et al., and R.S. Van Dyck, Jr. et al., in this volume) all rely on such doublets. There were three cases where we were able to identify 


\begin{tabular}{|c|c|c|c|}
\hline Nuclide & $\operatorname{mass}(u)$ & mass excess $(\mathrm{keV})$ & MistraL-AME95 (keV) \\
\hline${ }^{25} \mathrm{Ne}$ & $24.997707 \quad(32)$ & $-2136(30)$ & -77 \\
\hline${ }^{26} \mathrm{Ne}$ & 26.000461 & $429(30)$ & 0 \\
\hline${ }^{32} \mathrm{Mg}$ & $31.998858(113)$ & $\begin{array}{c}-1063(105) \\
\text { Table } 1\end{array}$ & -268 \\
\hline
\end{tabular}

New, Mistral mass measurements. In column 2: the mass (and error), in column 3: the mass excess (and error) and in column 3: the absolute deviations from the mass table [8].

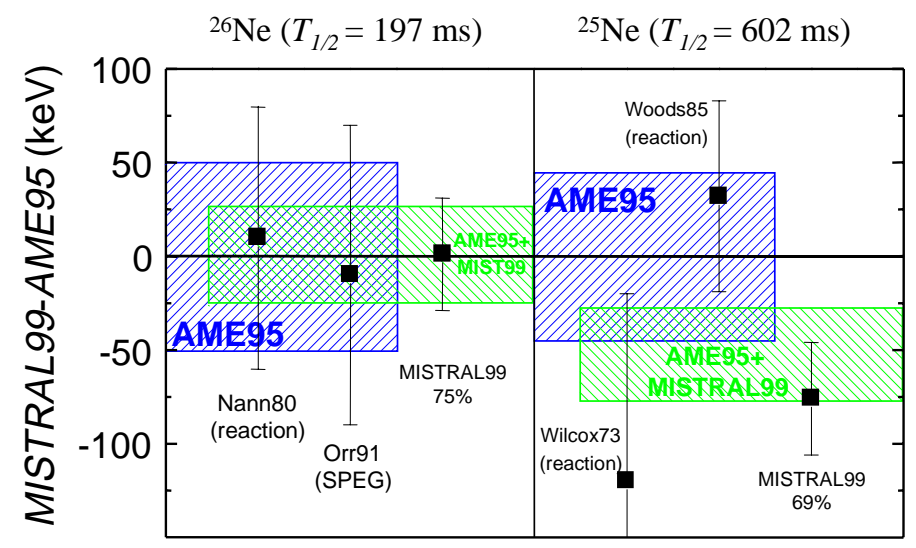

Figure 5. Absolute mass difference of MISTRAL results compared to the other results in [8] used to derive the recommended mass for ${ }^{26} \mathrm{Ne}$ (left) and ${ }^{25} \mathrm{Ne}$ (right). The influence of the MISTRAL values are also given.

isobaric pairs for measurement, shown in Fig.4 (right), and obtain an excellent measurement accuracy: $2 \cdot 10^{-7}$.

The final derived mass values for the three cases we were able to improve are listed in Table 1 along with their uncertainty and deviation from the mass table value [7].

An evaluation was performed for the results in Table 1 . In the case of the two neon isotopes, two values constituted the recommended mass in the table. For ${ }^{26} \mathrm{Ne}$, the reaction ${ }^{26} \mathrm{Mg}\left(\pi^{-}, \pi^{+}\right){ }^{26} \mathrm{Ne}$ was studied at LAMPF [9] and a direct measurement was made using SPEG at GANIL [10]. These values are compared to the MISTRAL value in Fig.5 (left) and can be seen to be perfectly compatible. The MISTRAL value now accounts for $75 \%$ of the weight.

The two data comprising the ${ }^{25} \mathrm{Ne}$ mass, shown in Fig.5 (right), come from reactions: ${ }^{26} \mathrm{Mg}\left({ }^{7} \mathrm{Li},{ }^{8} \mathrm{~B}\right){ }^{25} \mathrm{Ne}[11]$ and ${ }^{26} \mathrm{Mg}\left({ }^{13} \mathrm{C},{ }^{14} \mathrm{O}\right){ }^{25} \mathrm{Ne}$ [12]. Though the latter result has a smaller error bar due to the higher reaction cross-section and ${ }^{26} \mathrm{Mg}$ target enrichment, the MISTRAL value is closer to the former. However the new adjusted mass from the evaluation is still compatible with the old. Note the MISTRAL result carries almost $70 \%$ of the weight.

The result for ${ }^{32} \mathrm{Mg}$ is shown in Fig.6, again compared with the individual data that make up the recommended mass. All these data, from direct mea- 


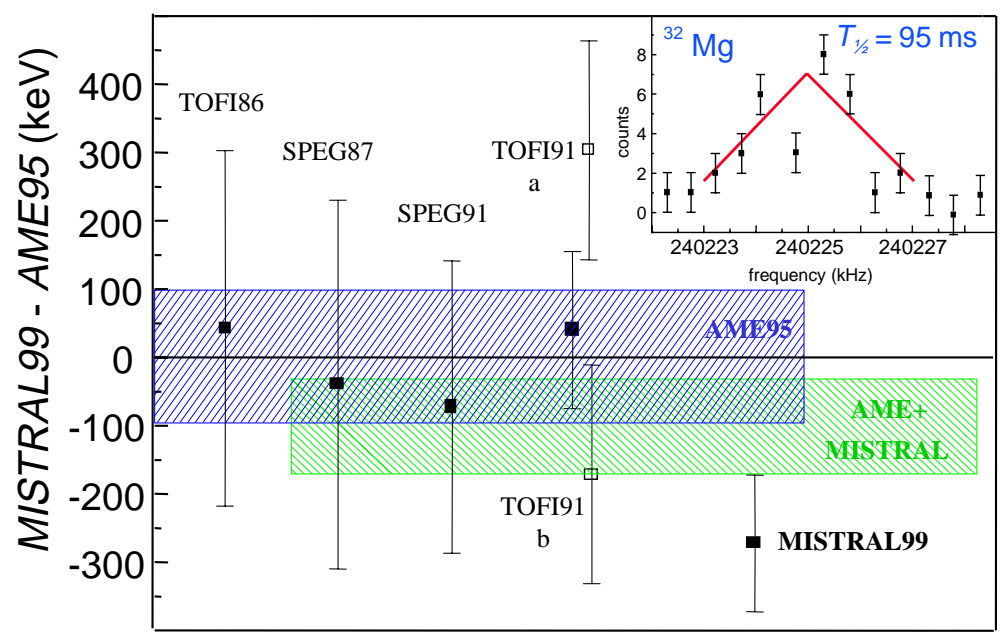

Figure 6. Absolute mass difference of MISTRAL result compared to the various results in [8] used to derive the recommended mass for ${ }^{32} \mathrm{Mg}$. The later TOFI value is in fact an average of two measurements as indicated in the figure. Inset: recorded mass peak with some 30 counts and a resolution of 80,000 .

surements with either TOFI [13,14] or SPEG [15,10], are compatible with each other and with the MISTRAL value with the exception of the later TOFI value [14] which is in fact an average of two runs, designated $a$ and $b$ in the figure. Fig.6(inset) shows the summed mass peak. The high resolving power of 80,000 was necessary to combat the high density of isobaric contamination and is reflected by the poor statistics, contributing an uncertainty of about $3 \cdot 10^{-6}$.

Despite an uncharacteristically large error bar, the MISTRAL mass, representing a $268 \mathrm{keV}$ overbinding with respect to the AME95 value, indicates the deformation effects thought to weaken the normally stabilizing shell closure at $N=20$ are more pronounced. The paper of F. Sarazin et al. in these proceedings treats this physics question.

\section{Conclusion}

The recent results show that in spite of high resolving power, the technique employed by MISTRAL of using harmonics is highly prone to the overwhelming isobaric contamination brought by the plasma ion source. Another run is planned using a laser ion source to avoid this.

On the other hand, there is absolutely no complication related to measuring the masses of nuclides having very short half-lives and provided that the calibration is done correctly, results of sufficient accuracy to improve mass data are obtained. This is demonstrated in the cases of ${ }^{25-26} \mathrm{Ne}$ and ${ }^{32} \mathrm{Mg}$. The latter nuclide, for which we find an overbinding compared to previous measurements, 
indicates a pronounced weakening of the normally stabilizing effect of a shell closure (here $N=20$ ).

The technique of using isobaric doublets has been shown to circumvent the calibration process that considerably lengthens data taking periods. It has since been used to measure the mass of the dripline nuclide ${ }^{74} \mathrm{Rb}$, a super-allowed beta emitter of importance for testing the electroweak sector of the standard model.

\section{Acknowledgements}

The results presented in this paper constitute part of the doctoral thesis of C. Monsanglant [7]. The authors would like to acknowledge the expert technical assistance of G. Le Scornet, M. Jacotin, J.-F. Képinski, G. Conreur from CSNSM, Orsay, M. Duma from IAP, Bucharest and G. Lebée from CERN. We thank Christoph Scheidenberger from GSI for his assistance during one of the experiments. This research project is funded by the IN2P3, France and some of the work at ISOLDE supported by the European RTD Programme "Access to Large-Scale Facilities".

\section{References}

[1] L.G. Smith, Phys. Rev.111 (1958) 1606

[2] M. de Saint Simon et al., Phys. Scripta T59 (1995) 406

[3] D. Lunney et al., Hyp. Interact. 99 (1996) 105

[4] A. Coc et al., Nucl. Instrum. Methods A 271 (1988) 512

[5] M. de Saint Simon, M. Jacotin and G. Lebée, internal CSNSM report 94-22 (1994), (available from [16])

[6] C.F. Toader, Doctoral thesis, Université de Paris Sud, Orsay, 1999 (available from [16])

[7] C. Monsanglant, Doctoral thesis, Université de Paris Sud, Orsay, 2000 (available from [16])

[8] G. Audi and A.H. Wapstra, Nucl. Phys. A 595 (1995) 409

[9] A. Nann et al., Phys. Lett. B96 (1980) 261

[10] N.A. Orr et al., Phys. Lett. B 258 (1991) 29

[11] K.H. Wilcox et al., Phys. Rev. Lett. 30 (1973) 866

[12] C.L. Woods et al., Nucl. Phys. A 437 (1985) 454

[13] D.J. Vieira et al., Phys. Rev. Lett. 57 (1986) 3253

[14] X.G. Zhou et al., Phys. Lett B 260 (1991) 285

[15] A. Gillibert et al., Phys. Lett. B 192 (1987) 39

[16] Atomic Mass Data Center (AMDC) http://csnwww.in2p3.fr/amdc/. 